\title{
Production de velours \\ chez le cerf rusa \\ (Cervus timorensis russa) \\ en Nouvelle-Calédonie: appréciation quantitative et qualitative du produit obtenu au stade d'aplatissement du merrain de la seconde branche
}

\author{
S. Le Bel ${ }^{1 *} \mathrm{Ph}$. Chardonnet ${ }^{2}$ M. Salas ${ }^{1}$
}

Mots-clés

Cervidae - Cervus timorensis russa Andouiller - Production animale Teneur en azote - Teneur en cendres Teneur en lipides - Teneur en eau N ouvelle-Calédonie.

\begin{abstract}
Résumé
Le velours de 13 cerfs rusa (Cervus timorensis russa) âgés de 3 à 5 ans a été récolté pendant deux campagnes de cinq mois après $63 \pm 2$ jours de pousse. Le poids de velours récolté (Pvt) a oscillé de 314 à 1225 g pour un poids moyen de $707 \pm 142 \mathrm{~g}$. Les facteurs saison de récolte et poids du cerf ont eu un effet significatif sur la production de velours. Au stade de récolte choisi, le velours avait la forme d'un $\mathrm{V}$ asymétrique dont la taille des branches était de $15,4 \pm 1,2$ et $24,0 \pm 1,5 \mathrm{~cm}$. Une équation de prédiction permet d'estimer la quantité de velours à récolter avant la coupe à partir des dimensions de celuici. Les analyses chimiques pratiquées montrent que le velours contient après dessiccation en moyenne 39 p. 100 de cendres, 57 p. 100 de matière protéique et 1 p. 100 de lipides. Les analyses par sections ont révélé que le velours est un produit hétérogène avec un gradient de la base vers les apex pour l'humidité, les teneurs en matière azotée et en matière grasse, et des apex vers la base pour la teneur en cendres brutes. Des équations de prédiction ont permis de calculer la teneur en matière grasse du produit à partir de la teneur des autres éléments. Par rapport au cerf élaphe (Cervus elaphus), le cerf rusa est handicapé par une saison de récolte étalée, un niveau de production limité à $1,2 \mathrm{~kg}$ par animal et un produit relativement pauvre en lipides.
\end{abstract}

\section{INTRODUCTION}

La dénomination « velours de cerf » s'applique au bois de cervidés récolté en cours de croissance, l'appellation velours venant de l'aspect velouté de la peau qui recouvre le bois. Cette production mésodermique fait l'objet d'une forte consommation en Asie du Sud-Est, notamment en Corée et à Hong Kong où il est utilisé pour la pharmacopée traditionnelle (12).

La demande en velours de cerfs rouges (Cervus elaphus) alimente un courant d'exportation soutenu des pays d'élevage du cerf vers

1. Cirad-emvt, BP 25, 98890 Païta, Nouvelle Calédonie

2. Cirad-emvt, Campus international de Baillarguet, BP 5035, 34032 Montpellier Cedex 1, France

* Auteur pour la correspondance

Email : lebel@cirad.nc les marchés asiatiques. A ce titre la Nouvelle-Zélande a exporté 200 tonnes de velours en 1994 pour une valeur marchande FOB de 60 millions de dollars nouveaux-zélandais (11).

Le velours des cerfs tropicaux (rusa, sambar, sika), traditionnellement consommé dans la zone indochinoise, fait l'objet d'un circuit de commercialisation informel de proximité. Le cerf rusa (Cervus timorensis russa), introduit en Nouvelle-Calédonie depuis 1870 , fournit ainsi un velours consommé par la communauté asiatique du Territoire. Bien qu'aucune production ne soit organisée, les élevages de cerfs sont régulièrement sollicités pour fournir des échantillons de velours aux pays asiatiques de la zone (Thaïlande, Malaisie, Hong Kong).

L'objectif de l'expérimentation était de caractériser le velours de cerf rusa sur le plan quantitatif en précisant notamment le poids de velours récoltable par cerf ainsi que ses dimensions. La valeur d'un velours étant notamment liée à sa teneur en lipides (6), l'expérimentation a consisté dans un second temps à préciser la composition chimique du velours de cerf rusa en examinant s'il était possible de prédire ce paramètre au moment de la récolte. 


\section{MATERIEL ET METHODES}

L'élevage expérimental de cerf rusa du Cirad-emvt est situé dans la station zootechnique de Port-Laguerre à une trentaine de kilomètres au nord de Nouméa, sur la façade ouest de la Grande Terre. La station bénéficie d'un climat de type tropical océanique, caractérisé par l'alternance irrégulière de trois saisons : chaude et humide (de janvier à mi-avril), fraîche (de mi-avril à août), puis sèche (de septembre à décembre).

Le cadre de l'expérimentation était une zone de pâturage améliorée de 10 ha, peu accidentée et non embuissonnée, et a concerné 14 cerfs rusa mâles adultes de 3 à 5 ans provenant du cheptel de Port-Laguerre.

\section{Observation de la croissance des velours}

Deux campagnes de récolte ont été menées, en 1993 puis en 1994. L'observation de la croissance des velours s'est effectuée à l'occasion de la première campagne de récolte.

En début d'année, les animaux, regroupés dans une parcelle dès janvier, ont été observés quotidiennement à la jumelle afin de noter pour chacun d'eux la date de chute du premier bois dur $(\mathrm{J} 0=$ début de pousse des velours) et la croissance des velours. La date de récolte des velours était fixée quand l'extrémité du merrain de la $2^{\mathrm{e}}$ branche s'aplatissait pour se diviser (figure 1). L'intervalle de temps entre la chute des bois précédents et le jour de la récolte était appelé durée de pousse (Dp) du velours, exprimée en jours.

\section{Collecte du velours}

Dès qu'un cerf se trouvait au stade de récolte défini précédemment, il était sorti du troupeau et immobilisé dans le piège à contention. La récolte s'est effectuée par ablation chirurgicale sous anesthésie locale (3 injections de 2,5 cc de Lurocaine ${ }^{\mathrm{ND}}$ autour du pivot) en coupant chaque velours à $2 \mathrm{~cm}$ au-dessus de la couronne.

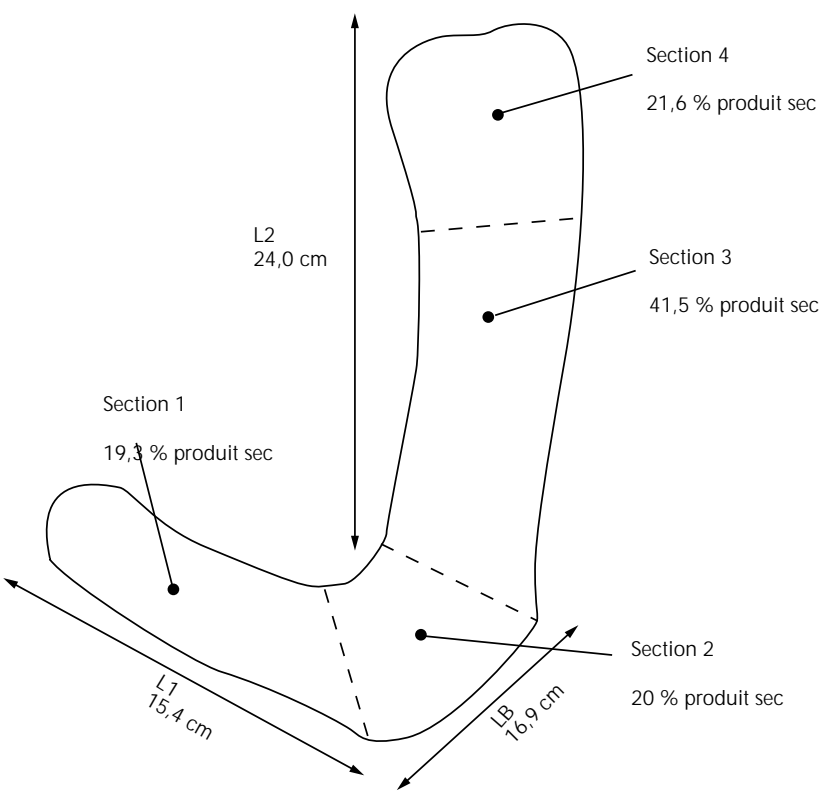

Figure 1 : stade de récolte et sections du velours.
Après le retrait du garrot, l'animal a été pesé, puis relâché. Les velours, une fois récoltés, ont été inclinés à $45^{\circ}$, l'extrémité vers le bas. Après identification, ils ont été congelés à $-18^{\circ} \mathrm{C}$.

\section{Mesures et analyses chimiques}

Les velours gauche et droit de chaque cerf ont été mesurés à l'aide d'un mètre à ruban : longueur de la première pointe (L1), longueur de la seconde pointe (L2), périmètre à la base (LB). Chaque velours a ensuite été pesé séparément $(\mathrm{Pv})$. Le poids total de velours récolté par cerf (Pvt) était la somme des poids du velours gauche et droit de l'animal (Pvt $=\mathrm{Pv}$ gauche $+\mathrm{Pv}$ droit $)$.

Chaque cerf a donc été caractérisé par un âge (Age), un poids vif $(\mathrm{Pvc})$, une saison de récolte $(\mathrm{Sr})$, une durée de pousse et un poids total de velours récolté.

Chaque velours a ainsi été caractérisé par un poids de velours $(\mathrm{Pv})$ et trois longueurs (LI, L2 et LB).

Les analyses chimiques ont porté sur les produits de la première campagne de récolte, soit 14 velours. Pour chaque animal, le velours droit a été subdivisé en quatre sections, numérotées de 1 à 4 (figure 1). Chaque section a été pré-dessiquée à $70{ }^{\circ} \mathrm{C}$, broyée et soumise aux analyses chimiques suivantes : taux d'humidité, teneur en cendres, teneur en matière azotée et teneur en lipides. Ce dernier composant a particulièrement retenu l'attention des auteurs dans la mesure où il a permis de qualifier un velours (6).

Les méthodes d'analyse chimique utilisées dans cet essai étaient les suivantes :

- détermination de l'humidité par dessiccation à $103{ }^{\circ} \mathrm{C}$ (NF V04401, janv. 68) ;

- détermination des cendres brutes (NF V04-404, janv. 68);

- détermination de l'azote total selon la méthode de Kjeldahl (NF V04-407, août 72). La teneur en matière protéique est obtenue en multipliant la teneur en azote par 6,25 ;

- détermination de la teneur en matière grasse totale par traitement à l'acide chlorhydrique et extraction à l'éther diéthylique (NF V04-402, janv. 68).

Les teneurs en humidité ont été exprimées en pourcentage de la matière fraîche. Les teneurs en cendres, matière protéique et matière grasse ont été exprimées en pourcentage de la matière sèche.

\section{Analyses statistiques}

Afin de comparer les quantités de Pvt obtenues selon les différentes classes d'âge, les saisons et en relation avec le Pvc des animaux, une analyse de covariance sur la variable Pvt en fonction des facteurs Age et Sr a été réalisée avec Pvc en covariable (procédure Manova SPSS/PC + version 5.0 (16)). Une analyse de variance (procédure Anova SPSS/PC + version 5.0 (16)) a été pratiquée sur la composition chimique du velours des différentes sections afin d'identifier l'existence de gradients de concentration. L'analyse a été réalisée sur l'arc sinus des valeurs obtenues.

Afin de mesurer l'effet des facteurs quantitatifs sur le poids de velours récolté par cerf, on procède à des tests de corrélation et à des régressions multiples pas à pas (procédure régression SPSS/PC + version 5.0 (16)). Le même type d'analyse est pratiquée sur la composition chimique du velours afin d'établir une équation de prédiction de la teneur en lipides. 


\section{RESU LTATS}

\section{Résultats des campagnes de récolte}

Les résultats des deux campagnes de récolte figurent dans le tableau I. Treize cerfs sur 14, âgés de 3 à 4 ans et pesant $78 \pm 4,5 \mathrm{~kg}$, ont été prélevés au cours de la première campagne, puis 9 , âgés de 4 à 5 ans et pesant $91 \pm 7,1 \mathrm{~kg}$, à l'occasion de la seconde campagne.

Le Pvt moyen récolté par cerf était de $582 \pm 99 \mathrm{~g}$ pour la première campagne et de $886 \pm 309$ g pour la seconde campagne. Le Pvt récolté pendant les deux campagnes était de $15,5 \mathrm{~kg}$, pour une valeur moyenne de $707 \pm 142 \mathrm{~g}$ (minimum $314 \mathrm{~g}$, maximum $1665 \mathrm{~g}$ ).

Pour les deux campagnes, 64 p. $100(\mathrm{n}=14)$ des récoltes ont été réalisées en saison chaude de janvier à mi-avril et 36 p. $100(n=8)$ en saison fraîche de mi-avril à août. Pour un stade donné (aplatissement du merrain de la seconde branche), le velours a été récolté après $63 \pm 2$ jours de croissance $(n=13$, minimum 57 jours, maximum 69 jours).

Les pesées des différentes sections (1 à 4) après dessiccation ont permis d'évaluer leur importance relative en pourcentage de produit sec (figure 1). Une analyse de variance (tableau II) a été réalisée sur l'arc sinus de cette variable en prenant comme facteur explicatif la portion de velours avec quatre modalités : les sections $1,2,3$ et 4. L'effet du facteur retenu était significatif ( $p<0,001$, 48 p. 100 de variance expliquée). Le calcul des moyennes par section a montré que le premier andouiller (section 1) et la base du velours (section 2) contenaient chacun $1 / 5$ du produit sec, respectivement 19,3 et 20,4 p. 100 du produit sec. La seconde pointe divisée en deux sections, section $3(41,5$ p. 100) et section 4 $(21,6$ p. 100) représentait les $3 / 5$ du poids sec d'un velours.

\section{Poids de velours récolté et facteurs de variation}

Les campagnes de récolte menées en 1993 et 1994 ont souligné la variabilité du poids de velours récolté par cerf. Deux types de facteurs étaient susceptibles d'expliquer cette variabilité : des facteurs liés à l'animal (âge, poids du cerfs) ou à la récolte (durée de pousse des velours au moment de la collecte, période de la récolte).

L'étude d'une matrice de corrélation (tableau III) entre les facteurs quantitatifs Age, Dp, Pvc et Pvt montre qu'il existe une série de corrélations significatives entre les facteurs Pvc, Pvt et Age. Le facteur Dp n'apparaît pas intéressant pour expliquer les variations observées.

L'âge et le poids du cerf étant partiellement liés par une régression significative $\left(\mathrm{Pvc}=8,8^{*} \mathrm{Age}+49,5 ; \mathrm{p}<0,01\right.$; écart-type résiduel $\left.=8,8 \mathrm{~kg} ; \mathrm{r}^{2}=0,30\right)$, une analyse de covariance portant sur les deux campagnes de récolte a été réalisée sur la variable Pvt en prenant comme facteurs explicatifs Age et $\mathrm{Sr}$ et Pvc comme covariable avec les modalités suivantes :

- facteur âge : 3 classes (3, 4 et 5 ans) ;

- facteur saison de récolte : 2 classes (saison chaude, saison fraîche).

L'hypothèse d'égalité entre les pentes des droites de régression des différentes modalités des facteurs explicatifs a été préalablement vérifiée. La régression entre Pvt et Pvc était significative $(\mathrm{t}=2,29, \mathrm{p}=0,04)$. Pvc apparaît (tableau IV) comme la principale variable expliquant significativement les variations de production observées. La saison a eu un léger effet significatif $(p<0,1)$ en faveur de la saison des pluies (tableau IV). Les différences de poids

Tableau I

Résultats des deux campagnes de récolte

\begin{tabular}{lccc} 
& $\begin{array}{c}\text { Première récolte } \\
\text { (effectif) }\end{array}$ & $\begin{array}{c}\text { Deuxième récolte } \\
\text { (effectif) }\end{array}$ & $\begin{array}{c}\text { Toutes les récoltes } \\
\text { (effectif, mini-maxi) }\end{array}$ \\
\hline Poids du cerf & $78,4 \pm 4,5$ & $91,0 \pm 7,1$ & $83,5 \pm 4,6$ \\
(Pvc en kg) & $(13)$ & $(9)$ & $(22,68-105)$ \\
Durée de pousse & $63 \pm 2$ & Pas d'observation & $63 \pm 2$ \\
(Dp en jours) & $(13)$ & $886 \pm 309$ & $(13,57-69)$ \\
Poids des velours & $582 \pm 99$ & $(9)$ & $707 \pm 142$ \\
(Pvt en g) & $(13)$ & & $(22,314-1665)$
\end{tabular}

\section{Tableau II}

Résultats de l'analyse de variance sur le pourcentage en produit sec avec comme facteur explicatif la zone de section

\begin{tabular}{lcccccc} 
Variable & Facteurs de variation & $\begin{array}{c}\text { Somme } \\
\text { des carrés }\end{array}$ & $\begin{array}{c}\text { Degré } \\
\text { de liberté }\end{array}$ & Carré moyen & Valeur de F & Signification \\
\hline Arc sinus \% produit sec & $\begin{array}{c}\text { Section } \\
\text { Variance résiduelle }\end{array}$ & 0,565 & 3 & 0,188 & 42,2 & $p<0,001$ \\
\% moyen en produit sec & Section 1 (effectif) & Section 2 (effectif) & Section 3 (effectif) & Section 4 (effectif) \\
\hline & $19,3 \pm 1,2(13)$ & $20,4 \pm 0,9(13)$ & $41,5 \pm 2,6(14)$ & $21,6 \pm 1,1(14)$
\end{tabular}


Tableau III

Matrice de corrélation entre les facteurs âge, poids vif du cerf, poids total de velours récolté et durée de pousse

\begin{tabular}{lllll} 
& Pvc (effectif) & Pvt (effectif) & D p (effectif) & Age \\
\hline Pvc & 1,00 & & & \\
Pvt & $0,67 * *(22)$ & 1,00 & 1,00 & \\
D p & 0,16 NS $(13)$ & 0,28 NS $(13)$ & 0,16 NS (13) & 1,00 \\
Age & $0,55 *(22)$ & $0,61 *(22)$ &
\end{tabular}

$* \mathrm{p}<0,01$

$* * \mathrm{p}<0,001$

NS : non significative, $p>0,05$

de velours récoltés selon l'âge s'expliquent donc en grande partie par les différences de poids entre les animaux. Le calcul des moyennes ajustées (tableau IV) montre que le Pvt oscillait de 612 à $771 \mathrm{~g}$ pour des animaux de 3 à 5 ans. L'effet saison de récolte s'est traduit par une diminution significative du poids de la récolte en saison fraîche de 20 p. 100 environ (773 g/623 g).

\section{Prédiction des quantités de velours récoltées}

\section{Mensuration des velours récoltés}

Les mensurations effectuées ont porté sur 37 des 44 velours récoltés pendant les deux campagnes (tableau V). Sept velours détériorés ont été retirés de l'effectif. Au stade d'aplatissement du merrain de la seconde branche, le velours récolté avait la forme d'un $\mathrm{V}$ asymétrique dont les deux branches mesuraient en moyenne respectivement $15,4 \pm 1,2$ et $24,0 \pm 1,5 \mathrm{~cm}$. La base du $\mathrm{V}$ avait une circonférence moyenne de $16,9 \pm 0,7 \mathrm{~cm}$, c'est-à-dire proche de la taille du premier andouiller (figure 1).

Afin d'apprécier l'effet âge sur la morphologie du velours au moment de la récolte, des analyses de variance ont été réalisées sur la variable mensuration en prenant comme facteur explicatif l'âge des cerfs selon trois classes : 3 , 4 et 5 ans (tableau VI). Si les sujets âgés de 5 ans ont effectivement présenté des velours un tiers (38 p. 100) plus grand qu'à 3 ans (LT), l'analyse statistique révèle que cet accroissement concerne surtout L1 qui double presque de taille (+ 82 p. 100) alors que L2 n'augmente que de 33 p. 100.

\section{Tableau V}

Mensurations des velours récoltés en $\mathrm{cm}(n=37)$

\begin{tabular}{lcc} 
Variable & Moyenne & Minimum/maximum \\
\hline L1 & $15,4 \pm 1,2$ & $2,0 / 22,5$ \\
L2 & $24,0 \pm 1,5$ & $16,0 / 33,0$ \\
LB & $16,9 \pm 0,7$ & $10,0 / 20,0$
\end{tabular}

\section{Prédiction du poids d'un velours en fonction de sa taille}

L'étude d'une matrice de corrélation entre Pv et L1, L2 et LB montre qu'il existe des corrélations significatives entre les $\mathrm{Pv}$ et les différents facteurs (tableau VII).

Une régression multiple pas à pas a été réalisée sur le $\mathrm{Pv}$ (en g) en prenant comme variables explicatives les mesures L1, L2 et LB (en $\mathrm{cm}$ ). La régression est significative et l'équation de prédiction du Pv s'exprime en fonction des trois mesures.

$\mathrm{Pv}=8 \mathrm{~L} 1+16 \mathrm{~L} 2+19 \mathrm{LB}(\mathrm{p}<0,001 ; \mathrm{n}=37$; écart-type résiduel $\left.=54 \mathrm{~g}, \mathrm{r}^{2}=0,84\right)$.

La valeur élevée du $\mathrm{r}^{2}$ signifie que l'équation est fiable pour prédire le poids d'un velours.

Cette équation s'avérant néanmoins difficile à utiliser sur le terrain, trois régressions ont été menées sur le facteur Pv en prenant comme mesure explicative chacune des mensurations (L1, L2, LB).

\section{Tableau IV}

Résultats de I'analyse de covariance sur la variable Pvt en prenant comme facteurs explicatifs l'âge du cerf, la saison de récolte et comme covariable le poids du cerf

$\begin{array}{lrrrrr} & \text { Somme des carrés } & \text { D egré de liberté } & \text { Carré moyen } & \text { Valeur de F } & \text { Signification } \\ \text { Régression (covariable) } & 159814 & 1 & 159814 & 5,21 & 0,04 \\ \text { Constante } & 16507 & 1 & 16507 & 0,54 & 0,48 \\ \text { Age } & 36624 & 2 & 18312 & 0,6 & 0,56 \\ \text { Saison } & 106316 & 1 & 106316 & 3,47 & 0,08 \\ \text { Intra-cellules } & 429331 & 14 & 30666 & & \end{array}$

Calcul des moyennes ajustées (g) selon l'âge et la saison de récolte

\begin{tabular}{lccc}
\hline Age de la récolte & 3 ans & 4 ans & 5 ans \\
& 612 & 712 & 771 \\
Saison de récolte & Saison des pluies & Saison fraîche & 623
\end{tabular}


Tableau VI

Résultats de I'analyse de variance sur les longueurs L1, L2 et LB avec I'âge comme facteur

\begin{tabular}{|c|c|c|c|c|c|c|}
\hline Variable & Source de variation & Somme des carrés & Degré de liberté & Carré moyen & Valeur de F & Signification \\
\hline L1 & $\begin{array}{l}\text { Effet âge } \\
\text { Variance résiduelle }\end{array}$ & $\begin{array}{l}287,4 \\
244,2\end{array}$ & $\begin{array}{r}2 \\
34\end{array}$ & $\begin{array}{r}143,7 \\
7,2\end{array}$ & 20,0 & $p<0,001$ \\
\hline L2 & $\begin{array}{l}\text { Effet âge } \\
\text { Variance résiduelle }\end{array}$ & $\begin{array}{l}167,3 \\
554,4\end{array}$ & $\begin{array}{r}2 \\
34\end{array}$ & $\begin{array}{l}83,7 \\
16,3\end{array}$ & 5,1 & $p<0,01$ \\
\hline LB & $\begin{array}{l}\text { Effet âge } \\
\text { Variance résiduelle }\end{array}$ & $\begin{array}{r}37,9 \\
136,3\end{array}$ & $\begin{array}{r}2 \\
34\end{array}$ & $\begin{array}{r}18,9 \\
4,0\end{array}$ & 4,7 & $p<0,05$ \\
\hline \multicolumn{7}{|c|}{ Calcul des moyennes ajustées (cm) selon l'âge des cerfs (effectif) } \\
\hline & 3 ans & 4 ans & & 5 ans & \multicolumn{2}{|c|}{$\begin{array}{c}\text { Variation entre } 3 \text { et } 5 \text { ans } \\
\text { en } \%\end{array}$} \\
\hline $\begin{array}{l}\text { L1 } \\
\text { L2 } \\
\text { LB }\end{array}$ & $\begin{array}{l}11,0(9) \\
20,6(9) \\
15,2(9)\end{array}$ & $\begin{array}{l}16,3(2 \\
24,8(2 \\
17,6(2\end{array}$ & & $\begin{array}{l}20,3(4) \\
27,4(4) \\
16,6(4)\end{array}$ & \multicolumn{2}{|c|}{$\begin{array}{r}+82 \\
+33 \\
+9\end{array}$} \\
\hline
\end{tabular}

\section{Tableau VII}

$M$ atrice de corrélation entre le poids d'un velours récolté $(P v)$ et ses mensurations ( $L 1, L 2, L B)$

\begin{tabular}{lllll}
$\begin{array}{l}\text { Corrélations } \\
\text { ( } \mathbf{n}=\mathbf{3 7})\end{array}$ & Pv & L1 & L2 & LB \\
\hline Pv & 1,00 & & & \\
L1 & $0,74 * *$ & & & \\
L2 & $0,82 * *$ & $0,62 * *$ & 1,00 & \\
LB & $0,61 * *$ & $0,47 * *$ & $0,31 \mathrm{NS}$ & 1,00 \\
$* * \mathrm{p}<0,001$ & & & & \\
NS : non significatif, $\mathrm{p}>0,05$ & & &
\end{tabular}

Les trois équations de régression étaient significatives $(\mathrm{p}<0,001)$. En raison de la valeur du $\mathrm{r}^{2}$ et de l'écart-type résiduel (Ectr), l'équation $n^{\circ} 2$ est à retenir en pratique pour évaluer avant la récolte le poids d'un velours au stade d'aplatissement du merrain de la seconde branche.

- $\mathrm{n}^{\circ} 1: \mathrm{Pv}=25 \mathrm{~L} 1-34(\mathrm{~F}=42, \mathrm{p}<0,001 ; \mathrm{n}=37 ; \mathrm{Ectr}=87 \mathrm{~g}$, $\left.\mathrm{r}^{2}=0,55\right)$;

$-\mathrm{n}^{\circ} 2: \mathrm{Pv}=23 \mathrm{~L} 2-215(\mathrm{~F}=70, \mathrm{p}<0,001 ; \mathrm{n}=37 ;$ Ectr $=75 \mathrm{~g}$, $\left.\mathrm{r}^{2}=0,67\right)$

- $\mathrm{n}^{\circ} 3: \mathrm{Pv}=35 \mathrm{LB}-254(\mathrm{~F}=21, \mathrm{p}<0,001 ; \mathrm{n}=37 ; \mathrm{Ectr}=103 \mathrm{~g}$, $\left.\mathrm{r}^{2}=0,37\right)$.

\section{Composition biologique du velours de cerf rusa}

\section{Composition globale}

Les résultats d'analyses globales pratiquées sur les velours montrent que le produit récolté contenait 32 p. 100 de matière sèche $(32,3 \pm 1,7 ; n=13)$. Après dessiccation, le produit sec contenait en moyenne 39 p. 100 de cendres, 57 p. 100 de matière protéique et 1 p. 100 de lipides (tableau VIII).

\section{Gradients de concentration}

Pour ce qui est des résultats par section de velours (1 à 4), des analyses de variances ont été réalisées sur l'arc sinus des variables

\section{Tableau VIII}

Composition globale du velours de cerf rusa en $\%$ de la matière sèche $(n=13)$

\begin{tabular}{lrc} 
& Moyenne & Minimum/maximum \\
\hline Teneur en cendres & $39,1 \pm 1,6$ & $33,0 / 43,0$ \\
Teneur en protéines & $56,9 \pm 1,5$ & $52,9 / 63,6$ \\
Teneur en lipides & $1,2 \pm 0,1$ & $0,9 / 1,5$
\end{tabular}

teneur en eau (Humidité), teneur en cendres brutes (Cendres), teneur en matière protéique (MP) et teneur en matière grasse (MG) en prenant comme facteur explicatif la portion de velours avec quatre modalités : les sections 1, 2, 3 et 4 .

L'analyse du tableau IX montre que le velours est un produit hétérogène sur le plan qualitatif. Il existe des gradients (figure 2), d'une part, de la base vers les apex pour l'humidité, les teneurs en matière azotée et en matière grasse et, d'autre part, des apex vers la base pour la teneur en cendres brutes.

La partie la plus riche en lipides, donc la plus intéressante sur le plan pharmacologique pour la préparation de pantocrine (extrait alcoolique de velours), est le dernier tiers de la $2^{\mathrm{e}}$ pointe où la teneur en lipides est en moyenne de 2,13 $\pm 0,11$ p. 100 .

\section{Prédiction de la teneur en matière grasse}

La teneur en lipides étant un élément intéressant à prédire (fabrication de pantocrine), une série de tests de corrélation et de régression a été réalisée sur ce paramètre (teneur globale et par section) en prenant comme facteurs explicatifs les données obtenues au moment de la récolte : âge et poids du cerf, durée de croissance du velours, dimensions et poids du velours. La conduite des tests n'a pas permis de prédire de manière significative la teneur en lipides d'un velours ou d'une des sections au moment de la récolte. 


\section{Tableau IX}

Résultats de l'analyse de variance sur la composition chimique avec comme facteur explicatif la zone de section

\begin{tabular}{|c|c|c|c|c|c|c|c|c|c|}
\hline \multirow{2}{*}{$\begin{array}{l}\text { Variable } \\
\text { Arc sinus \% humidité }\end{array}$} & \multicolumn{2}{|c|}{$\begin{array}{l}\text { Facteurs } \\
\text { de variation }\end{array}$} & \multirow{2}{*}{$\begin{array}{c}\begin{array}{c}\text { Somme } \\
\text { des carrés }\end{array} \\
0,531\end{array}$} & \multirow{2}{*}{$\begin{array}{c}\begin{array}{c}\text { Degré } \\
\text { de liberté }\end{array} \\
3\end{array}$} & \multicolumn{2}{|c|}{ Carré moyen } & \multicolumn{2}{|c|}{ Valeur de F } & \multirow{3}{*}{$\begin{array}{c}\text { Signification } \\
p<0,001\end{array}$} \\
\hline & \multirow{2}{*}{\multicolumn{2}{|c|}{$\begin{array}{l}\text { Section } \\
\text { Variance résiduelle }\end{array}$}} & & & \multirow{2}{*}{\multicolumn{2}{|c|}{$\begin{array}{l}0,177 \\
0,005\end{array}$}} & \multirow{2}{*}{\multicolumn{2}{|c|}{36,6}} & \\
\hline & & & 0,242 & 50 & & & & & \\
\hline \multirow[t]{2}{*}{ Arc sinus $\%$ cendres } & \multirow{2}{*}{\multicolumn{2}{|c|}{$\begin{array}{l}\text { Section } \\
\text { Variance résiduelle }\end{array}$}} & 0,436 & 3 & \multirow{2}{*}{\multicolumn{2}{|c|}{$\begin{array}{l}0,145 \\
0,001\end{array}$}} & \multirow{2}{*}{\multicolumn{2}{|c|}{105,2}} & \multirow[t]{2}{*}{$p<0,001$} \\
\hline & & & 0,069 & 50 & & & & & \\
\hline Arc sinus & \multirow{2}{*}{\multicolumn{2}{|c|}{$\begin{array}{l}\text { Section } \\
\text { Variance résiduelle }\end{array}$}} & 0,297 & 3 & \multirow{2}{*}{\multicolumn{2}{|c|}{$\begin{array}{l}0,099 \\
0,001\end{array}$}} & \multirow{2}{*}{\multicolumn{2}{|c|}{75,6}} & \multirow[t]{2}{*}{$p<0,001$} \\
\hline$\%$ matière protéique & & & 0,066 & 50 & & & & & \\
\hline \multirow{2}{*}{$\begin{array}{l}\text { Arc sinus } \\
\% \text { matière grasse }\end{array}$} & \multirow{2}{*}{\multicolumn{2}{|c|}{$\begin{array}{l}\text { Section } \\
\text { Variance résiduelle }\end{array}$}} & 0,030 & 3 & \multirow{2}{*}{\multicolumn{2}{|c|}{$\begin{array}{l}0,010 \\
<0,001\end{array}$}} & \multirow[t]{2}{*}{38,1} & \multirow{2}{*}{\multicolumn{2}{|c|}{$p<0,001$}} \\
\hline & & & 0,013 & 50 & & & & & \\
\hline \multicolumn{2}{|c|}{ Composition moyenne par section } & \multicolumn{2}{|c|}{ Section 1 (effectif) } & \multicolumn{2}{|c|}{ Section 2 (effectif) } & \multicolumn{2}{|c|}{ Section 3 (effectif) } & \multicolumn{2}{|c|}{ Section 4 (effectif) } \\
\hline \multicolumn{2}{|l|}{$\begin{array}{l}\text { Humidité } \\
\text { en } \% \text { de produit frais }\end{array}$} & \multicolumn{2}{|c|}{$65,1 \pm 0,6(13)$} & \multicolumn{2}{|c|}{$60,6 \pm 0,6(13)$} & \multicolumn{2}{|c|}{$65,6 \pm 1,3(14)$} & \multicolumn{2}{|c|}{$75,9 \pm 1,0(14)$} \\
\hline \multicolumn{2}{|l|}{$\begin{array}{l}\text { Cendres } \\
\text { en } \% \text { de matière sèche }\end{array}$} & \multicolumn{2}{|c|}{$38,2 \pm 1,2(13)$} & \multicolumn{2}{|c|}{$47,8 \pm 1,0(13)$} & $42,6 \pm$ & $(14)$ & 25, & $1 \pm 0,9(14)$ \\
\hline $\begin{array}{l}\text { Matière protéique } \\
\text { en } \% \text { de matière sèche }\end{array}$ & & 57 & $\pm 1,2(13)$ & $49,4 \pm 0,9$ & & $53,7 \pm$ & 14) & 68, & $7 \pm 0,9(14)$ \\
\hline $\begin{array}{l}\text { Matière grasse } \\
\text { en } \% \text { de matière sèche }\end{array}$ & & 1,4 & $\pm 0,14(13)$ & $0,76 \pm 0,0$ & & $0,90 \pm$ & $(14)$ & 2,1 & $3 \pm 0,11(14)$ \\
\hline
\end{tabular}

Des analyses similaires ont été menées afin d'évaluer la possibilité d'estimer rapidement la teneur en lipides à partir d'autres composants. Pour l'analyse globale du velours (tableau X), il existe une corrélation significative $(\mathrm{p}<0,01)$ avec la teneur en matière

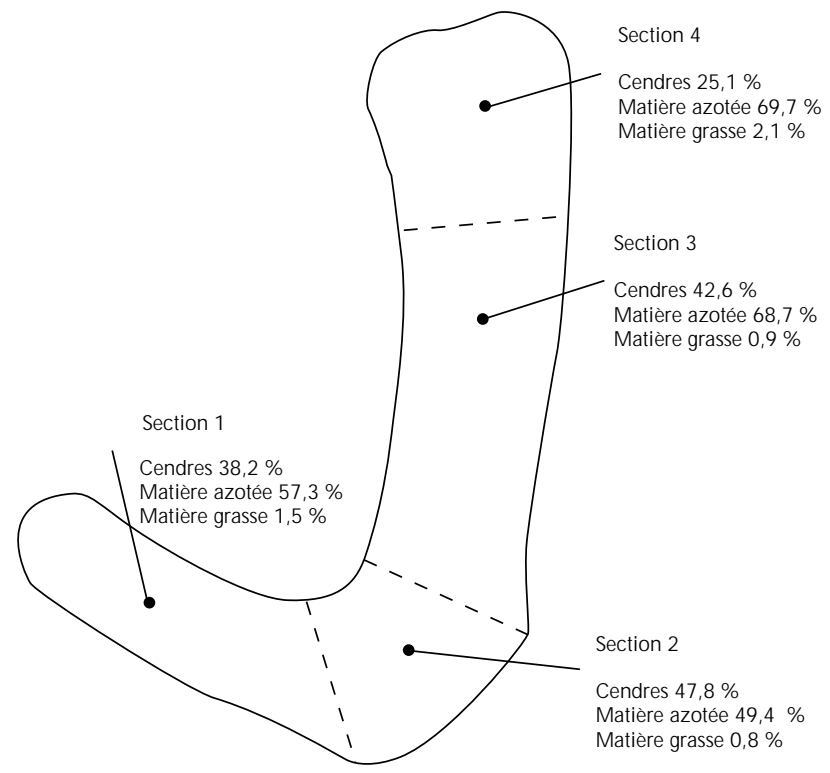

Figure 2 : gradients de concentration. protéique. Pour la section $\mathrm{n}^{\circ} 1$, une corrélation significative ( $p<0,001)$ existe entre la teneur en matière grasse et la teneur en cendres (tableau XI). L'équation de régression obtenue dans le cadre des analyses globales a permis d'estimer la teneur en lipides (MG) en fonction de la teneur en matière protéique (MP) (équation $\left.n^{\circ} 1\right)$. Celle obtenue avec les résultats de la première section a permis d'estimer le taux de matière grasse $(\mathrm{MG})$ à partir de la teneur en cendres (MX) (équation $\mathrm{n}^{\circ} 2$ ).

Equation $\mathrm{n}^{\circ} 1$ (composition globale) $: \mathrm{MG}=(0,045 \times \mathrm{MP})-1,35$ $\left(\mathrm{p}<0,01 ; \mathrm{n}=13 ; \operatorname{Ectr}=0,14, \mathrm{r}^{2}: 0,45\right)$.

Equation $\mathrm{n}^{\circ} 2\left(\right.$ section $\left.\mathrm{n}^{\circ} 1\right): \mathrm{MG}=(-0,11 \mathrm{x} \mathrm{MX})+5,79$ $\left(\mathrm{p}<0,001 ; \mathrm{n}=13 ; \operatorname{Ectr}=0,17, \mathrm{r}^{2}=0,90\right)$.

Sur le plan pratique, l'équation $\mathrm{n}^{\circ} 1$ présente peu d'intérêt dans la mesure où il est plus rapide et moins coûteux de doser la teneur en lipides qu'en protéines.

\section{Tableau X}

Matrice de corrélation entre les teneurs des différents composants du velours. Analyse globale $(n=13)$

\begin{tabular}{lrcll} 
Corrélations & MS & Cendres & MP & MG \\
\hline MS & 1,00 & & & \\
Cendres & 0,30 & 1,00 & & \\
MP & $-0,39$ & $-0,94^{* *}$ & 1,00 & \\
MG & 0,01 & $-0,59$ & $0,67^{*}$ & 1,00
\end{tabular}

MS : matière sèche ; MP : matière protéique ; MG : matière grasse $* \mathrm{p}<0,01 ; * * \mathrm{p}<0,001$ 
Tableau XI

Matrice de corrélation entre les teneurs des différents composants du velours. Analyse de la section $n^{\circ} 1(n=13)$

\begin{tabular}{lrccc} 
Corrélations & Humidité & Cendres & MP & MG \\
\hline Humidité & 1,00 & & & \\
Cendres & $-0,03$ & 1,00 & & \\
MP & 0,20 & $-0,82^{* *}$ & 1,00 & \\
MG & $-0,10$ & $-0,95^{* *}$ & $0,70 *$ & 1,00
\end{tabular}

MP : matière protéique ; MG : matière grasse

$* \mathrm{p}<0,01 ; * * \mathrm{p}<0,001$

\section{DISCUSSION}

\section{Production de velours, aspect quantitatif}

La méthode de récolte du velours décrite est un procédé couramment utilisé dans les élevages de cerfs élaphes néo-zélandais au point qu'il figure dans une charte précisant les modalités de cette production (17). L'auteur indique qu'il est possible d'utiliser deux méthodes de contention (chimique ou mécanique) et deux procédés d'anesthésie locale, une méthode tronculaire bloquant les nerfs infratrochléaire et zygomaticotemporal ou une méthode circulaire autour du pédicule. Ce dernier procédé d'anesthésie s'avère plus efficace (14)

La récolte du velours au moment de l'aplatissement du merrain de la seconde branche est un stade de récolte classiquement retenu en Australie chez le cerf rusa (Cervus timorensis russa, Cervus timorensis moluccensis) (8). Il correspond à l'obtention d'un produit satisfaisant le marché asiatique, notamment celui de Taïwan (2).

La récolte du velours est effectuée en moyenne 63 jours après que le cerf ait épaulé. English, cité par Chardonnet (2), rapporte une durée de pousse de 55 à 60 jours chez le cerf rusa. La récolte se situe classiquement 60 jours après la chute des anciens bois chez le cerf élaphe d'après Cowie (4) et 45 jours chez le daim (Dama dama) d'après English (5). L'originalité de l'espèce étudiée se situe dans la longueur de la période de récolte (2). Elle a été de cinq mois dans le cadre de cette étude et de deux mois chez le cerf élaphe (10). L'explication de ce phénomène est liée à la physiologie de ce cervidé tropical dont le rut est peu saisonnier (1). Cette particularité apparaît comme une contrainte chez les éleveurs de cerfs calédoniens peu habitués à manipuler fréquemment leur cheptel (13)

Les quantités de velours récoltées par cerf ont oscillé de 300 à $1200 \mathrm{~g}$ en produit frais pour un poids moyen de $707 \mathrm{~g}$. Chez le cerf rusa, English, cité par Chardonnet (2), rapporte des niveaux de production de 0,6 à $1,0 \mathrm{~kg}$ pour des mâles de 2 à 3 ans et de $1,5 \mathrm{~kg}$ pour des individus plus âgés. Au même stade de coupe, soit un velours de catégorie A (grille qualitative néo-zélandaise), le cerf rusa a une production comparable à celle du cerf sika (Cervus nippon), avec $0,5-1 \mathrm{~kg}$ de velours, et se situe à un niveau intermédiaire entre le cerf élaphe, avec 4,5-5 kg de velours, et le daim, avec 300-400 g de velours $(4,6,9)$.

Dans cette étude, les facteurs poids du cerf et saison de récolte, à un moindre degré, ont eu un effet significatif sur la production de velours. Des études similaires chez le cerf élaphe $(4,9)$ et le cerf sika (18) ont abouti aux mêmes conclusions. Fennessy et Suttie (9) évoque néanmoins chez cette dernière espèce l'influence du régime alimentaire sur les quantités de velours récoltées.

\section{Production de velours, aspect qualitatif}

La division du velours en quatre morceaux (1 à 4) montre après dessiccation que trois des quatre sections $\left(\mathrm{n}^{\circ} 1\right.$ : la première branche $; n^{\circ} 2$ : la base ; $n^{\circ} 4$ : l'apex) ont représenté chacune de 19 à 21 p. 100 du produit sec. La quatrième section $\left(n^{\circ} 3:\right.$ les deux tiers de la seconde branche) a représenté 39 p. 100 du produit sec. Ce type de découpage pratiqué sur le velours de cerf élaphe (6) fournit des sections plus homogènes avec trois morceaux sur quatre représentant un tiers du produit sec, l'apex du velours ne représentant que 2,5 à 3 p. 100 du produit sec.

Des analyses globales pratiquées par Fennessy et Duncan (8) sur trois velours de cerf rusa australien donnent des résultats proches de ceux de cette étude avec 37,9 p. 100 de cendres, 8,6 p. 100 d'azote et 1,2 p. 100 de lipides. Le velours de cerf rusa calédonien apparaît un peu plus riche en matière protéique avec 9,1 p. 100 d'azote (teneur en matière protéique $=6,25 \mathrm{x}$ azote Kjeldahl).

L'analyse chimique du velours des autres espèces de cervidés $(6,7,8)$ montre qu'à un stade de récolte équivalent (catégorie $\mathrm{A})$, les velours des cerfs élaphe et sika sont plus riches en lipides (cerf élaphe : 2 à 5,5 p. 100 de lipides; cerf sika : 2,2 p. 100 de lipides) et moins riches en protéines $(48,8$ à 52,5 p. 100) et en cendres (33,1 à 36,9 p. 100). Ces résultats signifient que le velours de cerf rusa est un produit relativement peu intéressant pour la production de pantocrine, extrait alcoolique de velours (6).

Pour ce qui est des résultats d'analyses pratiquées par section, Fennessy $(6,7)$ et Fennessy et Duncan (8) aboutissent aux mêmes conclusions avec le cerf élaphe : le velours est un produit hétérogène avec un gradient de concentration de la base vers l'apex. Cette dernière section est la partie la plus riche en lipides et la moins minéralisée. Ce phénomène reflète le processus de croissance et de différenciation du velours avec minéralisation graduelle du produit à partir du pivot osseux (15). Chez le cerf élaphe, il existe une équation de prédiction de la teneur en lipides en fonction de la teneur en cendres (pourcentage de lipides $=(7,42$ $\pm 0,064)-0,145$ p. 100 cendres ; $r^{2}=0,21$, Ectr $\pm 0,50$; d'après Fennessy et Duncan (8)). Dans cette étude, une équation du même type permet de prédire le taux de matière grasse du premier andouiller (pourcentage de lipides $=5,79$ - (0,11 x p. 100 cendres $)$ ).

Au stade de récolte fixé dans cette étude, il est impossible de prédire la qualité chimique du velours de cerf rusa, notamment sa richesse en lipides. Chez le cerf rouge où il existe une situation similaire, Fennessy (7) a déterminé le stade optimal de récolte en superposant les courbes de variation des teneurs en cendres et en lipides du velours en fonction du temps. L'intersection des deux courbes détermine le stade de croissance du velours où la quantité récoltée est maximale avec un taux de lipides satisfaisant les utilisateurs. Cette voie d'approche mériterait d'être testée avec le cerf rusa afin de déterminer le stade de récolte où la qualité chimique $\mathrm{du}$ velours se rapprocherait de celle obtenue avec le cerf rouge.

\section{- CON CLUSIO N}

Au stade d'aplatissement du merrain de la seconde branche, un cerf rusa adulte peut fournir un maximum de 1000 à $1200 \mathrm{~g}$ de produit frais, la variabilité des niveaux de production observés dépendant du poids du cerf. 
La méthode de récolte chirurgicale avec anesthésie circulaire autour de chaque pivot et contention mécanique paraît adaptée au cerf rusa et permettrait de mener des campagnes de récolte à plus grande échelle. Néanmoins, la durée de la période de récolte qui excède cinq mois est une contrainte sérieuse à l'organisation de campagnes de récolte en Nouvelle-Calédonie.

L'équation établie entre le poids et les mensurations des velours permet de prédire avant la coupe les quantités récoltées et de sélectionner les animaux les plus productifs.

Comme pour les autres espèces de cervidés, le velours de cerf rusa est un produit contenant en moyenne 1 p. 100 de lipides, 39 p. 100 de cendres et 57 p. 100 de protéines. Une des caractéristiques de ce produit est de présenter un gradient de concentration de la base vers l'apex, cette dernière partie étant la plus riche en lipides et la moins minéralisée. Avec une teneur en matière grasse de 1 p. 100, le velours du cerf rusa apparaît moins intéressant que celui du cerf élaphe pour extraire de la pantocrine.

Des travaux portant sur l'évolution de la composition chimique du velours au cours de sa croissance s'avèrent nécessaires pour déterminer un stade de récolte plus intéressant.

\section{Remerciements}

Nous remercions chaleureusement M. J.-C. Hurlin, technicien production animale, Cirad-emvt, Nouvelle Calédonie, pour l'aide qu'il nous a apportée à l'occasion de la récolte des velours.

\section{BIBLIO GRAPH IE}

1. BIANCHI M., 1993. Reproduction du cerf rusa de Java (Cervus timorensis russa) en Nouvelle-Calédonie. N ouméa, Nouvelle Calédonie, Cirad-emvt, 41 p. (Rapport)

2. CHARDONNET Ph., 1988. Etude de faisabilité technique et économique de l'élevage de cerfs en Nouvelle-Calédonie. MaisonsAlfort, France, Cirad-iemvt, $282 \mathrm{p}$.

3. CHARDONNET Ph., GRIMAUD P., AGATHE-NERINE S., 1990 Commercial rusa deer production in New Caledonia. In: 2 nd int. Wildl. Ranching Symp., Edmonton, Alberta, Canada, June 4-11, 1990. Paris, France, IGF, $12 \mathrm{p}$.
4. COW IE J., 1989. Stag breeding. In: Proc. Deer course for veterinarians No. 6, July 1989. Queenstown, NZ, Deer Branch NZ Vet. Assoc., p. 129-131.

5. ENGLISH A.W., 1990. Fallow deer production research in Australia. In: Proc. Deer course for veterinarians No. 7, July 1990. Auckland, NZ, Deer Branch NZ Vet. Assoc., p. 128-134.

6. FEN N ESSY P.F., 1989. Pharmacology of velvet. In: Proc. Deer course for veterinarians N o. 6, July 1989. Q ueenstown, NZ, Deer Branch NZ Vet. Assoc., p. 96-103.

7. FEN NESSY P.F., 1991. Velvet antler: the product and pharmacology. In: Proc. Deer course for veterinarians N 0. 8, May 1991. Sydney, Australia, Deer Branch NZ Vet. Assoc., p. 169-180.

8. FEN NESSY P.F., DUNCAN S.J., 1992. Comparative composition of velvet antler. In: Proc. Deer course for veterinarians No. 9, June 1992. Methven, NZ, Deer Branch NZ Vet. Assoc., p. 26-35.

9. FEN NESSY P.F., SUTTIE J.M., 1985. Antler growth: nutritional and endocrine factors. Biology of deer production. R. Soc. N.Z., Bull., 22: 239-250.

10. GIB, 1991. Velveting guidelines from the GIB. In: The New Zealand deer breeding and management manual. New Zealand deer development council, p. 77-86.

11. HUTCHING B., 1995. Growth in velvet exports tracked. The Deer farmer, 0 ct. (126): 20-23.

12. KONG Y.C., BUT P.P.H., 1985. Deer. The ultimate medicinal animal (antler and deer parts in medecine). Biology of deer production. R. Soc. N.Z., Bull. 22: 311-324.

13. LE BEL S., 1993. L'élevage du cerf rusa en Nouvelle-Calédonie. Bilan et perspectives. Nouméa, Nouvelle Calédonie, Cirad-emvt Nouvelle-Calédonie, 26 p. (Coll. Etudes et synthèses)

14. MATTHEWS L.R., IN GRAM J., COOK C., BREM NER K., KIRTON P., 1992. Induction and assessment of velvet analgesia. In: Proc. Deer course for veterinarians No. 9, June 1992. Methven, NZ, Deer Branch NZ Vet. Assoc., p. 69-76.

15. MUIR P.D., SYKES A.R., BARRELL G.K., 1985. Mineralisation during antler growth in red deer. Biology of deer production. R. Soc. N.Z., Bull. 22: $251-254$

16. SPSS, 1992. SPSS/PC+ Base system user's guide, version 5.0. Chicago, IL, USA, M arijia J. N orusis/SPSS Inc., 910 p.

17. WILSO N P.R., 1989. Velvet harvesting: moral, ethical and legal aspects. In: Proc. Deer course for veterinarians No. 6, July 1989. Q ueenstown, NZ, Deer Branch NZ Vet. Assoc., p. 104-117.

18. ZHOU S., WU S., 1985. Velvet antler production of Sika Deer. Biology of deer production. R. Soc. N.Z., Bull. 22: 282.

Accepté le 17.08 .98 


\section{Summary}

Le Bel S., Chardonnet Ph., Salas M. Rusa deer (Cervus timorensis russa) velvet production in New-Caledonia: qualitative and quantitative results obtained at the stage of division of the main beam

The velvet of 13 rusa deer (Cervus timorensis russa) 3 to 5 years of age were harvested during two five-month periods after a $63 \pm 2$ days' growth. The velvet weight ranged from 314 to $1225 \mathrm{~g}$ with an average weight of $707 \pm 142 \mathrm{~g}$. The harvest season and live weight factors had a significant effect on velvet production. At the harvest chosen stage, the velvet had asymmetrical V-shape branches, $15.4 \pm 1.2 \mathrm{~cm}$ and 24.0 $\pm 1.5 \mathrm{~cm}$ long. The quantity of velvet to be harvested can be estimated via a prediction equation based on velvet size prior to cutting. Chemical analyses show that on average velvet contains after drying 39\% ashes, $57 \%$ nitrogen and $1 \%$ lipids. Section analyses revealed that velvet is heterogeneous with a gradient from the base to the tips for humidity, nitrogen and fat, and from the tips to the base for ashes. Fat content can be calculated based on the content of other elements by means of prediction equations. Compared to the red deer (Cervus elaphus), the rusa deer is disadvantaged by spread-out harvest seasons, $1.2 \mathrm{~kg}$ top production level per animal and a relatively low fat content.

Key words: Cervidae - Cervus timorensis russa - Antler Animal production - Nitrogen content - Ash content - Lipid content - Moisture content - New Caledonia.

\section{Resumen}

Le Bel S., Chardonnet Ph., Salas M. Producción de pelusa de cuerno en el ciervo rusa (Cervus timorensis russa) en Nueva Caledonia: estimación cuantitativa y cualitativa del producto obtenido en el estadio plano de la segunda rama del cuerno

Se recolectó la pelusa de 13 ciervos rusa (Cervus timorensis russa), de 3 a 5 años de edad, durante dos campañas de cinco meses, a los $63 \pm 2$ días de crecimiento. El peso de la pelusa obtenida (Pvt) osciló entre 314 y 1225 g para un peso medio de $707 \pm 142 \mathrm{~g}$. Los factores de época de colecta y peso del ciervo tuvieron un efecto significativo sobre la producción de pelusa. En el estadío de recolección seleccionado, la pelusa tuvo forma de $\mathrm{V}$ asimétrica, con un tamaño de ramas de $15,4 \pm 1,2$ y $24,0 \pm 1,5 \mathrm{~cm}$. Mediante una ecuación de predicción se estimó la cantidad de pelusa por recolectar antes de la poda, a partir de las dimensiones de este cuerno. Los análisis químicos efectuados, demuestran que la pelusa del cuerno contiene, después del secado, un promedio de $39 \%$ de cenizas, $57 \%$ de materia proteica y $1 \%$ de lípidos. Los análisis por sección revelaron que la pelusa es un producto heterogéneo, con un gradiente de la base hacia el apex para la humedad, el contenido de materia nitrogenada y de materia grasa; y del apex hacia la base para la tenencia en cenizas brutas. Las ecuaciones de predicción permitieron el cálculo del contenido en materia grasa del producto a partir del contenido de otros elementos. En comparación con el ciervo «elafo» (Cervus elaphus), el ciervo rusa se encuentra en desventaja por una estación de recolta evidente, un nivel de producción limitado a 1,2 kg por animal y un producto relativamente pobre en lípidos.

Palabras clave: Cervidae - Cervus timorensis russa - Asta Producción animal - Contenido de nitrógeno - Contenido de ceniza - Contenido de lípidos - Contenido de humedad Nueva Caledonia. 